\title{
EXPLOTACIÓN DE RECURSOS NATURALES Y CONFLICTO EN COLOMBIA
}

\author{
Luis Eduardo Sandoval* \\ Margarita Marín** \\ Ana María Almanza***
}

$\mathrm{D}$ iversos autores estudian los conflictos por recursos naturales en Colombia, en particular en el agro y la minería. Bejarano (1986) señala que los problemas de violencia social e inseguridad en regiones ganaderas se enfrentaron con aumentos del pie de fuerza, mejor equipamiento de policías y militares y formación de grupos paramilitares. Arias e Ibáñez (2012) indican que en municipios de alto conflicto el uso de la tierra se trasladó a cultivos transitorios y ganadería. Goebertus (2008) muestra que la transición del banano al aceite de palma en la zona bananera ha influido en el conflicto armado, sobre todo en el desplazamiento forzado, por el cobro de extorsiones, la menor intensidad de mano de obra y la seguridad alimentaria, y la usurpación de tierras por actores armados ilegales.

En minería, Monte et al. (2014) muestran que las comunidades cimarronas han sido afectadas por los cambios en sus formas tradicionales de producción y sus costumbres sociales, por cuenta de prácticas empresariales en Cerrejón. Torres (2014) comprueba que en algunos proyectos mineros existen conflictos étnicos, sociales,

* Profesor asociado, Departamento de Economía, Universidad Militar Nueva Granada, Bogotá, Colombia, [luis.sandoval@unimilitar.edu.co].

** Profesora del Departamento de Matemáticas, Universidad Sergio Arboleda, Bogotá, Colombia, [margarita.marin@correo.usa.edu.co]

**** Asistente de investigación, Facultad de Ciencias Económicas, Universidad Militar Nueva Granada, Bogotá, Colombia, [ana.almanzav@gmail.com]. Este artículo es parte del proyecto INV ECO 2071 Relación entre recursos naturales y condiciones socioeconómicas con la dinámica de la violencia en Colombia, 2000-2014, financiado por la Vicerrectoría de Investigaciones de la Universidad Militar Nueva Granada. Fecha de recepción: 27-09-2016, fecha de modificación: 21-03-2017, fecha de aceptación: 04-09-2017. Sugerencia de citación: Sandoval, L. E.; M. Marín y A. M. Almanza. (2017). Explotación de recursos naturales y conflicto en Colombia, Revista de Economía Institucional 19(37), 201-225. Dor: https://doi.org/10.18601/01245996.v19n37.11 
ambientales y violentos. Rodríguez et al. (2014) señalan que Pacific Rubiales Energy y Pacific Coal Resources ha vulnerado los derechos laborales, afectado la seguridad humana en las zonas de explotación y provocado conflictos ambientales y sociales y culturales en las comunidades aledañas a las explotaciones. Los informes del Cinep de Rivera et al. (2012) muestran los problemas sociales generados en Colombia por las políticas de extracción minera en la última década.

Estos resultados son un punto de partida para entender los problemas ligados a la explotación minero-energética. Este trabajo se concentra en los conflictos en torno a la explotación de recursos mineros y las consecuencias ambientales, culturales, sociales y de violencia de la minería legal e ilegal en las regiones. En línea con la medición de la relación entre conflicto y recursos naturales abordada por la literatura ${ }^{1}$, este artículo hace un aporte cuantitativo y multidimensional al problema, caracterizando la conflictividad por la explotación de carbón, petróleo, oro y ferroníquel mediante un índice.

Los minerales que se consideran representan más del 90\% de las explotaciones y exportaciones minerales del país ${ }^{2}$, de modo que son de gran importancia para su política extractiva. Aunque la explotación de esmeraldas también provoca conflictos (Leiteritz et al., 2009), no es objeto del presente estudio.

Se busca establecer si la mayor disposición de recursos naturales aumenta el conflicto social en los municipios. Concretamente, el índice estudia interacciones entre amenazas y vulnerabilidades que identifican riesgo por disposición de recursos naturales, mostrando si una mayor (menor) disposición implica un menor (mayor) desarrollo económico que provoca y prolonga formas de violencia y conflictos a nivel municipal.

El artículo se divide en cuatro secciones. En la primera se revisa la bibliografía sobre la relación entre conflictos y recursos naturales, así como las dimensiones del conflicto que estudia la literatura y las que aquí se proponen. En la segunda sección se describen las hipótesis, la formación del indicador y el método para construirlo. En la cuarta se

\footnotetext{
${ }^{1}$ Por ejemplo, Bannon y Collier (2003), Humphreys (2005), Collier (2007) y Brunnschweiler y Bulte (2009).

2 El Ministerio de Minas y Energía clasifica los minerales en cinco tipos: metálicos (metales preciosos, siderúrgicos, básicos, ligeros, nucleares y especiales), industriales (potasio, azufre, cuarzo, trona, sal, amianto, talco, feldespato y fosfatos), de construcción (arena, grava, áridos, arcillas, caliza y equistos), gemas (diamantes, rubíes, zafiros y esmeraldas) y combustibles (carbón, lignito y turba). Si bien, según el Código de Minas, los minerales no incluyen el petróleo ni el gas, son combustibles fósiles que la literatura incluye en la discusión sobre extracción de minerales. Dada la coyuntura y los conflictos que ha provocado en el país y a nivel mundial, aquí se incluye el petróleo como un mineral.
} 
presentan los resultados y sus implicaciones. La sección final expone las conclusiones.

\section{ESTADO DEL ARTE}

\section{RECURSOS NATURALES Y CONFLICTO}

Aunque existen numerosos trabajos sobre la relación entre conflictos y recursos naturales, esta sección se concentra en la literatura sobre la relación entre conflictos violentos o armados y recursos naturales.

Collier (2007) señala que la riqueza natural contribuye a la trampa del conflicto. Los recursos naturales y el superávit de estos reducen el crecimiento de un país porque los ingresos que proporcionan no compensan la pérdida de crecimiento. Hay casos donde el crecimiento se compromete a causa de los recursos naturales porque la población vive de la renta que generan. En países donde esa renta es media se presentan fuertes auges y descalabros económicos porque los ingresos por recursos naturales son volátiles, dificultan la gobernabilidad y generan crisis.

Humphreys (2005) afirma que el impacto de los recursos naturales en los conflictos es independiente de la fortaleza del Estado. E1 conflicto es originado por la producción en periodos anteriores y no por la producción futura. Por tanto, la existencia de estos recursos se relaciona con guerras de corta duración, que suelen finalizar con una victoria militar, cuando la oferta de recursos naturales se ve amenazada. En la literatura sobre conflictos y explotación petrolera, Morrison (2010) indica que la relación entre producción de petróleo y comienzo de conflicto desaparece cuando la capacidad estatal es fuerte, mientras que la tendencia de la producción de petróleo a estabilizar los regímenes políticos desaparece en un contexto de instituciones débiles.

Bannon y Collier (2003) concluyen que los países en desarrollo tienen mayor riesgo de conflictos violentos y mala gobernabilidad si son dependientes de bienes primarios, siendo la explotación legal o ilegal de los recursos la que financia los conflictos. Aunque la riqueza de recursos naturales no es la única causa del conflicto ni lo hace inevitable, aumenta el riesgo de que este surja o se prolongue. Brunnschweiler y Bulte (2009) encuentran que la dependencia de recursos naturales es endógena al conflicto pero que este aumenta la dependencia de la extracción de recursos naturales, mientras que la abundancia de recursos naturales se relaciona con una baja probabilidad de inicio de guerra. Omeje (2006) muestra que la conflictividad 
es ocasionada por la corrupción en la contratación pública, más que por la corrupción en contratación privada.

En cuanto a la relación entre conflictos y explotación carbonífera se destacan los trabajos sobre conflictos entre industrias y comunidades, y sobre conflictos por costos de mano de obra y tratos laborales, de Scott et al. (1963), Lewis (1987) y Morrice y Colagiuri (2013). Pero no hay estudios cuantitativos que evalúen tal relación.

En el caso de la explotación aurífera, los estudios de Mensah y Okyere (2014), Özen y Özen (2011) y Schueler et al. (2011) relacionan la explotación de oro y los conflictos, desde una perspectiva cuantitativa, principalmente en temas políticos, ambientales, sociales.

En cuanto a los conflictos por explotación de ferroníquel, se destaca el trabajo de Rettberg (2014), que hace un estudio por regiones, recursos y tipos de conflicto en Colombia, enfocándose en el único municipio donde se realiza esta extracción. Muestra que no hay control estatal y que, aunque la explotación de ferroníquel no explica la presencia de grupos armados, sí ayuda a justificar parcialmente la duración del conflicto.

\section{RECURSOS MINERO-ENERGÉTICOS Y CONFLICTO EN COLOMBIA}

La conflictividad identificable y transversal a los recursos naturales se encuentra en la dicotomía entre explotación legal e ilegal. De acuerdo con la Ley 685 de 2001, la minería ilegal es una actividad exploratoria desarrollada sin el título minero vigente. Sin embargo, también existe la minería artesanal, de carácter legal, pero la ley no puede distinguir claramente y así pierde efectividad para combatir la ilegalidad (Procuraduría General de la Nación, 2011; Ministerio de Minas y Energía, 2014).

La minería informal y la minería ilegal en Colombia tienen las siguientes implicaciones: 1) evaden sistemas de control sobre recursos naturales renovables y no renovables (Corporación Autónoma Regional del Valle, 2010); 2) no pagan las cargas tributarias establecidas por el Estado; 3) ocasionan daños irreversibles a ecosistemas protegidos (Procuraduría General de la Nación, 2011); 4) no cumplen los requisitos mínimos de seguridad laboral, protección social, salud ocupacional y normatividad de contratación infantil; y 5) son una importante fuente para la captación de recursos que carece de un control efectivo del Estado, que pueden ser utilizados para financiar actividades ilegales (ввс, 2010; de la Corte, 2011).

Aunque se reconoce que la minería informal causa más conflictos que la minería legal, en la literatura también hay referencias a 
conflictos en las explotaciones mineras consideradas. Por ejemplo, en algunas zonas petroleras las compañías han instalado estructuras paraestatales de poder, $\mathrm{y}$ algunas adquirieron derechos sobre tierras y recursos en las zonas. E1 caso más extremo se observó en la Concesión Barco, donde los pequeños propietarios de predios aledaños al oleoducto se convirtieron en usurpadores de estas tierras (Jiménez y Sideri, 1985).

Además, la explotación minera crea enclaves de producción que debido a los altos salarios desestabilizan la economía local, afectan la producción agraria y generan desempleo urbano (Avellaneda, 1998). Igualmente, provoca conflictos con las instituciones y poblaciones locales por daños ambientales e invasión de territorios étnicos, y propicia la violación de los derechos humanos como respuesta a la resistencia de comunidades indígenas y poblaciones campesinas (Cusaria y Alfonso, 2004).

Como ilustración de estos problemas, las referencias al conflicto se remontan a las primeras concesiones del gobierno para explotar petróleo, cuando la persecución a los indígenas yariguíes y bari los llevó a la extinción (Posso et al., 2013). En los años cuarenta la Concesión de Mares desalojó a la fuerza a los colonos y expropió a los indígenas zenúes del Catatumbo (Cusaria y Alfonso, 2004). La formación de enclaves impulsó una urbanización desproporcionada que produjo contaminación, hacinamiento, segmentación social y violencia (Posso et al., 2013). Finalmente, en la literatura se reconoce que Ecopetrol nace de una huelga contra la Concesión de Mares (ibíd.) y de los bajos ingresos recibidos por el gobierno, debido a las grandes concesiones otorgadas a las empresas norteamericanas (Hernández, 2010).

Otro ejemplo de afectación a indígenas es el de las explotaciones de la Texas Petroleum y Ecopetrol en Putumayo. Allí, los pueblos inga, kofán, siona, eitoto y koreguaje fueron reducidos a una sexta parte y perdieron cerca del $94 \%$ de su territorio luego de 30 años de explotación (Cusaria y Alfonso, 2004).

En esta misma línea se encuentra la disputa entre la etnia u'wa y la Occidental de Colombia por la explotación de hidrocarburos en el Piedemonte Llanero, que culminó con la suspensión de la licencia ambiental de exploración, pues la actividad atentaba contra la identidad étnica y cultural de la comunidad indígena (Hernández, 2010). En la explotación del pozo de Casabel, en Yondó, se destruyeron hectáreas de ciénaga por el vertimiento de agua contaminada en el proceso de deshidratación y por la filtración de los pozos petroleros (Cusaria y Alfonso, 2004). 
En los pozos de Cicuco y Boquete, el pasivo ambiental generado por las explotaciones de Colpet y Ecopetrol llevó a conflictos que no solo llevaron cerca de la extinción a la pesca artesanal, sino que mostraron la debilidad de la legislación ambiental por la imposibilidad estatal para hacerla cumplir (ibíd.). Otros ejemplos de daños ambientales se observan en los pozos Cuaguaqui-Teran, explotados por la Texas Petroleum Company, y San Francisco, por Ecopetrol y Shell-Hocol.

Posso et al. (2013) señalan que la actividad petrolera afecta las condiciones sociales y económicas de las comunidades cercanas a esta explotación, específicamente por los siguientes factores: incrementos acelerados en la población, incremento de desempleo, incremento en precios, desplazamiento de comunidades y afectaciones al ordenamiento territorial, entre otros.

En lo que respecta al conflicto armado, se reconoce que la inconformidad por la política petrolera de principios del siglo pasado fue uno de las razones del origen de la guerrilla del elN (Cusaria y Alfonso, 2004). Estas conflictividades no son exclusivas de la explotación petrolera y pueden ser extrapoladas a la explotación de otros recursos mineros.

En cuanto al problema social, Rudas (2014) señala altos niveles de pobreza en zonas con explotación minera, comparado con zonas de otro tipo de explotación. El autor muestra que la minería de carbón es un mal motor en la generación de empleo, pues contribuye solo con cerca del 1\% del empleo local. Igualmente, Rudas y Espitia (2013) indican que la remuneración en minería es baja, pues por cada 100 pesos de excedente bruto de trabajo se remunera un máximo de 40 pesos, comparado con un pago de 90 en agricultura y 80 en industria. Adicionalmente, la minería desplaza estas actividades e incrementa el desempleo local y disminuye la remuneración local (Rueda, 2014).

Monte et al. (2014) analizan el conflicto económico, social y cultural creado en comunidades afro de La Guajira por la explotación carbonífera. Las comunidades del departamento señalan que las prácticas empresariales en El Cerrejón han implicado la apropiación de sus tierras por parte de las empresas, así como cambios en las formas tradicionales de producción y mutación de costumbres sociales. A esto se unen la denuncia de las comunidades locales de afectaciones ambientales a las cuencas acuíferas y los problemas de salud generados por el polvillo de carbón. En este último aspecto la situación es más incierta: Rudas (2014) indica que no existe bibliografía sobre impactos de la explotación de carbón en el suelo y en el agua. Y no 
son muy claros los planes de mitigación ambiental y las afectaciones a la salud humana por parte de muchas de las empresas.

Se reconoce además que existen conflictos sociales, económicos, ambientales y violentos por la explotación de oro. Por ejemplo, existe un gran debate en torno a la explotación de la mina La Colosa, en la zona de Cajamarca (Quindío), pues afecta directamente la zona protegida del Valle del Cocora (El Espectador, 2014) e incluso, en 2017, la comunidad, por medio de una consulta popular, dijo que no deseaba la ejecución de proyectos mineros en el municipio.

Además, las empresas AngloGold Ashanti Colombia S.A. y B2Gold Corp., y Gran Colombia Gold en Antioquia, han reconocido que sus explotaciones producen impactos ambientales en cuanto a contaminación auditiva, contaminación de cuencas, afectación de flora y fauna, y desplazamiento de la comunidad, en zonas cercanas al lugar de explotación (AngloGold Ashanti, 2015).

De acuerdo con Rodríguez et al. (2014), los municipios de Segovia, Remedios y Zaragoza, que tienen explotaciones mineras legales e informales, registran niveles de mercurio mil veces más elevados que los que se consideran seguros para la salud humana.

Respecto a conflictos políticos, en la zona del Amazonas las comunidades han hecho fuertes denuncias de actos de corrupción. Por ejemplo, se sobornó a miembros de las comunidades indígenas para que interpusieran una acción de tutela por la creación del Parque Natural Yaigojé-Apaporis en las zonas de explotación de la empresa Cosigo Resources, arguyendo la falta de consulta previa (Fundación Ideas para la Paz, 2014).

En cuanto a conflictos violentos, los grupos de narcotráfico usan los recursos procedentes de la explotación del oro para financiar actividades ilegales tanto de grupos armados al margen de la ley (Ramírez y Muñoz, 2012) como de lavado de activos (Mazo, 2013). Adicionalmente, existe evidencia de que la entrada de empresas a sitios con minería artesanal tradicional ha generado protestas sociales que no siempre son pacíficas (Rettberg y Riomalo, 2014).

El caso del ferroníquel en Colombia es especial, pues este mineral se produce únicamente en el municipio de Montelíbano (Córdoba), en Cerro Matoso, la mina a cielo abierto más grande del continente y la cuarta más grande del mundo (Semana, 2012). Existen denuncias de afectaciones al medio ambiente debido a la cantidad de escombros arrojados por la operación minera (ibíd.). Además, los trabajadores han reportado problemas laborales en la mina y declarado huelgas ( $E l$ Tiempo, 2016a, 2016b). La comunidad ha señalado que las autoridades 
locales son corruptas, y sustenta su denuncia en la poca inversión social en el municipio, a pesar de las billonarias regalías recibidas (Semana, 2015), de las cuales el municipio ha generado una fuerte dependencia financiera (De la Hoz, 2009a). Igualmente, en la zona se ha reportado presencia de grupos armados ilegales, así como de cultivos ilícitos, que provocaron desplazamiento forzado (De la Hoz, 2009b).

A pesar de todos los problemas mencionados en esta sección, es importante reconocer que algunas empresas en los últimos años han venido realizando esfuerzos, en coordinación con el gobierno, con el fin de desarrollar estrategias de mitigación -en algunas ocasiones más allá de las que exige la ley-con el fin de disminuir sus afectaciones a las comunidades.

\section{EL CONFLICTO Y SUS DIMENSIONES}

El conflicto en la minería puede ser visto desde perspectivas sociales, ambientales y políticas, entre otras. Así, por ejemplo, para Bebbington (2009) los conflictos en minería están asociados a disputas ambientales, vistas como confrontaciones entre modelos de sostenibilidad. Por el contrario, Akpalu y Parks (2007) analizan el problema de la explotación de oro desde un enfoque económico-ambiental. Estos autores consideran que los problemas generados sobre los bosques tropicales pueden ser internalizados mediante un impuesto al ingreso de la explotación aurífera.

Whiteman y Mamen (2002) analizan la relación entre justicia, minería y comunidades indígenas en Panamá, encontrando que existe negligencia hacia la dimensión cultural, lo que exacerba la conflictividad.

De Echave et al. (2009) encuentran que las grandes conflictividades en el sector minero se deben a problemas de gobernabilidad, gestión ambiental y participación ciudadana. Twose (1991) y Fairhead (2001) señalan que el problema de los recursos naturales es cíclico pues causa conflictos sociales, que ocasionan marginalización de la población rural, inestabilidad social y política, desplazamiento y migraciones que a su vez vuelven a causar conflicto. Torres (2014), que analiza la conflictividad en La Colosa y La Toma desde una perspectiva social y ambiental, encuentra una expansión de los conflictos étnicos y de violencia con participación de grupos armados legales e ilegales que forzaron el desplazamiento de las comunidades.

Teniendo en cuenta lo anterior, se considera que la aproximación a la conflictividad minera debe realizarse desde un enfoque multidimensional en los siguientes cuatro ejes: 
1. Ambiental: considera efectos ambientales como deforestación; captación y contaminación de aguas; vertimientos líquidos, sólidos y gaseosos; deslizamientos; contaminación auditiva, afectación de los ecosistemas, flora y fauna.

2. Social: considera problemas que causan afectaciones de carácter social o económico a la comunidad, como: problemas de migración e incremento de la población, desempleo local, problemas de abastecimiento en servicios públicos, incremento de los precios, problemas de Рот, problemas de tenencia de la tierra y problemas relacionados con seguridad social, prestaciones sociales y salud ocupacional.

3. Violencia: considera problemas asociados a conflictos armados y violencia urbana, tales como: militarización de zonas, conflictos de salud pública (alcohol, drogas y tráfico sexual), violaciones de DDHH y del DIH, conflicto armado, y financiación de actividades criminales.

4. Cultural: considera afectaciones a costumbres y tradiciones sociales, económicas y políticas de comunidades protegidas, como afectación de las costumbres indígenas ancestrales, afectación de las formas de producción tradicional, del uso del suelo, y de las formas de relación social.

La descripción y las fuentes de datos de cada uno de estos ejes se presentan en el cuadro 2 .

\section{HIPÓTESIS, FORMACIÓN DEL INDICADOR Y METODOLOGÍA ESTADÍSTICA}

\section{Hipótesis de ESTUdIO}

De acuerdo con ciertas teorías de conflictividad y recursos naturales, la intensidad y la ubicación de los hechos de conflicto en el país se relaciona con la riqueza natural y mineral disponible. En efecto, las luchas por la obtención de beneficios económicos y sociales derivados de la explotación de recursos naturales prolongan los conflictos entre los diversos actores involucrados (personas, empresas, Estado, grupos ilegales). A pesar de esto, diversas posturas señalan que en el caso colombiano estas no se cumplen en su totalidad, sino que existen efectos diferenciadores entre regiones.

Teniendo en cuenta las dimensiones del conflicto propuestas a la luz de las teorías estudiadas, se parte de las siguientes hipótesis para el caso colombiano:

Hipótesis 1. No existe una relación directa entre explotación minera y conflictividad. De acuerdo con la tesis de Bannon y Collier (2003), no es cierto que en todos los municipios donde se extraigan minerales de 
manera legal o informal se presenten conflictos ambientales, sociales, culturales o de violencia. Por el contrario, se considera que habrá municipios donde el conflicto es más preponderante que en otros, y por tanto las políticas públicas deben ser diferenciadas.

Hipótesis 2. Existe un efecto diferenciado en la relación entre recursos naturales y conflictividad. Las conflictividades generadas por cada uno de los recursos naturales son diferentes, aunque en algunos momentos se traslapan. Así, las conflictividades asociadas a la explotación aurífera son diferentes a las asociadas a la carbonífera y petrolífera, y por tanto las políticas públicas deben ser diferenciadas.

Hipótesis 3. Aunque existe relación entre la debilidad estatal y las conflictividades en zonas de recursos naturales, esta no es una de las causas preponderantes. Humphreys (2005) señala que no existe relación entre debilidad estatal y conflictividad: existen algunas zonas con poca presencia estatal sin que esto haya generado grandes conflictos relacionados con la minería. De igual manera, existen zonas con débil presencia estatal en la que se han generado muchos conflictos.

Hipótesis 4. El desarrollo social es diferenciado en las regiones de explotación minera. Existe la percepción de que las regiones con explotación de recursos mineros, aunque reciben grandes ingresos del gobierno, tienen un pobre desempeño en materia social. Sin embargo, este efecto es diferente según la región.

\section{FORMACIÓN DEL INDICADOR}

Los indicadores propuestos permiten relacionar conflictividad y explotación por tipo de recurso mineral predominante en las regiones, de una manera ponderada. La construcción del indicador parte de conocer la métrica de las variables que tienen las dimensiones. De este modo se pueden agrupar variables con métricas distintas y se normalizan en una misma dimensión (Causey, 1969). En tal sentido se plantea un índice agregado de conflicto multidimensional según las dimensiones ambiental, social, violencia y cultural.

A partir de las hipótesis planteadas, el indicador debe mostrar: para la hipótesis 1 , la posibilidad de conflicto según condiciones regionales; para la hipótesis 2, los diferentes tipos de conflictos; para la hipótesis 3, la presencia estatal, y para la hipótesis 4, las diferencias en el nivel de desarrollo regional.

Dado lo anterior, el indicador propuesto considera:

1. $\Gamma\left(\mathrm{X}_{\mathrm{i}, \mathrm{d}, \mathrm{t}}\right)=\sum_{\mathrm{i}=1}^{\mathrm{n}} \mathrm{f} \tau(\mathrm{x})$ combinaciones lineales de las variables en cada dimensión 
2. $\varphi\left(\mathrm{X}_{\mathrm{i}, \mathrm{d}, \mathrm{t}}\right)=\mathrm{f}\left(\mathrm{IC}_{\mathrm{a} ; \mathrm{i}, \mathrm{t}}, \mathrm{IC}_{\mathrm{s}, \mathrm{i}, \mathrm{t}}, \mathrm{IC}_{\mathrm{vi}, \mathrm{t}, \mathrm{IC}}, \mathrm{IC}_{\mathrm{ci}, \mathrm{t}}\right)$, tipos de conflictos (ambiental, social, violento, cultural)

3. $\mathrm{V}\left(\mathrm{X}_{\mathrm{i}, \mathrm{d}, \mathrm{t}}\right)=\frac{1}{\mathrm{n}} \sum_{\mathrm{i}=1}^{\mathrm{n}}\left(\mathrm{x}_{\mathrm{i}, \mathrm{t}}-\underline{\mathrm{x}}\right)^{2}$ diferencia en variables de desarrollo regional

4. $\mathrm{w}(\mathrm{d}, \mathrm{i}, \mathrm{t})$, variables de presencia estatal

Así, resulta el indicador:

$\operatorname{ICM}_{\mathrm{i}, \mathrm{d}, \mathrm{t}}=\operatorname{Max}\left(\mathrm{X}_{\mathrm{i}, \mathrm{d}, \mathrm{t}}\right)=\frac{\Gamma\left(\mathrm{X}_{\mathrm{i}, \mathrm{d}, \mathrm{t}}\right) \varphi\left(\mathrm{X}_{\mathrm{i}, \mathrm{d}, \mathrm{t}}\right) \mathrm{w}(\mathrm{d}, \mathrm{i}, \mathrm{t})}{\varphi\left(\mathrm{X}_{\mathrm{i}, \mathrm{d}, \mathrm{t}}\right)}, \mathrm{ICM}_{\mathrm{i}, \mathrm{d}, \mathrm{t}} \in[0,1]$

Este indicador agrupa las hipótesis de estudio y propone una medida compuesta a partir de la disponibilidad de recursos minerales en las regiones, los tipos de conflictos, la varianza en términos de desarrollo municipal, y la presencia del Estado.

Con el índice resultante se muestra si existe alguna paradoja por disposición de recursos minerales en las diferentes regiones del país a partir de múltiples dimensiones de conflicto que perduran en el tiempo y pueden retardar el crecimiento regional por las capturas de rentas generadas por los recursos naturales. Se muestra, para el caso colombiano, que existe una relación entre el recurso natural y el riesgo de conflicto.

\section{MÉTOdo ESTAdístico}

En este trabajo se utiliza el método del análisis factorial múltiple (AFM) junto con métodos de clasificación para elaborar un índice de probabilidad de conflicto municipal, considerando las cinco dimensiones antes mencionadas.

Se empleó este método porque permite asignar pesos a los individuos y a las variables con los mismos datos, lo que reduce el sesgo del investigador en la construcción del índice. Además, permite combinar múltiples dimensiones de análisis, por lo que el estudio de los conflictos se puede realizar a nivel agregado, para cada mineral y para cada dimensión de interés.

Aunque el método permite determinar correlaciones entre variables y no su causalidad, es una de las mejores maneras de analizar las relaciones entre un gran número de variables. Además, es uno de los métodos por excelencia para construir indicadores de riesgo, $\mathrm{y}$ ha sido utilizado para hacer mediciones y clasificaciones en temas de paz y riesgo humanitario ${ }^{3}$.

\footnotetext{
${ }^{3}$ Ver, p. ej., los índices de riesgo humanitario de Naciones Unidas o el Índice
} de Paz Global. 
Finalmente, se recurre a métodos de clasificación, con el fin de crear categorías de análisis que faciliten la interpretación entre los municipios, pues al existir alrededor de 500 municipios con explotación minera, su comparación mediante el uso directo del índice dificultaría la validación (o no) de las hipótesis de trabajo.

En el análisis de componentes principales (ACP) la información se organiza en una tabla rectangular: las columnas representan variables de valores numéricos continuos y las filas representan a los individuos sobre los cuales se han medido las variables. El objetivo es la búsqueda de un sub espacio que se encuentre lo más cercano posible al conjunto original de datos. Esto se logra minimizando la distancia entre el sub espacio buscado y los datos originales (nube de datos), es decir, maximizando la varianza (Lebart et al., 2006). Para esto es necesario obtener los valores y vectores propios de la matriz de covarianza de los datos (ACP no normado) o de la matriz de correlaciones de los datos (ACP normado).

Con ello se obtiene cada uno de los componentes principales (o ejes factoriales), de manera que el primer factor surge de la combinación de las variables usando como coeficientes el primer vector propio asociado al valor propio más alto. La notación usual es $\operatorname{ACP}(\mathrm{X}, \mathrm{D}, \mathrm{M})$, donde $\mathrm{X}$ representa la tabla de datos, mientras que $\mathrm{D}$ y $\mathrm{M}$ representan las matrices de pesos para los individuos y las variables que, de no existir, se consideran como de igual peso (Escoffier y Pagès, 1992).

Para construir un índice con este método se realiza un ACP, asegurando que haya una alta correlación con alguno de los ejes (la mayor parte del tiempo se utiliza el primero), y se selecciona el vector propio asociado. El peso de cada variable en el índice corresponde a la participación del coeficiente asociado a la variable (es decir, el componente del vector propio asociado a la variable) en la suma total de coeficientes. El índice es la suma ponderada de estas variables de acuerdo con su peso.

El método anterior se puede extender para considerar agrupaciones de variables, es decir, cuando pertenecen a un grupo (o dimensión) y se desea asignar pesos a las variables y a los grupos. Para ello se utiliza el AFM, en el que se aplica un ACP equilibrando el peso de los grupos (Le Dien y Pagès, 2003a). Es decir, se da una ponderación a los grupos de variables, de modo que la matriz $\mathrm{M}$ se modifica, tal que: $\mathrm{M}=\frac{1}{\lambda_{1}^{j}} \mathrm{I}_{\mathrm{p}}$ 
donde $\lambda_{1}^{j}$ representa la varianza más grande del grupo $\mathrm{j}$; $\mathrm{I}_{\mathrm{p}}$, la matriz idéntica de orden p, X y D se mantiene igual a la del ACP (Pagès, 2004). Así, los grupos tienen una ponderación equilibrada en la construcción del primer eje, aunque un grupo con mayor número de variables contribuirá más a los componentes (Le Dien y Pagès, 2003b).

Partiendo de los resultados anteriores, para construir el índice se debe seleccionar el vector propio del eje de interés y re ponderarlo por la varianza de cada grupo. Una vez re ponderado, el índice se construye de igual manera que en el ACP. Una vez obtenidos los índices, se estandarizan usando la transformación:

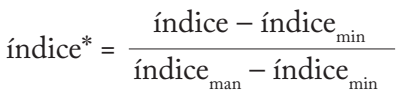

Donde índice $_{\min }$ indica el valor mínimo del índice e índice ${ }_{\max }$ su valor máximo. Una limitación de este método es que los resultados, y los pesos de las variables y de los grupos, se ven influenciados por las variables elegidas para construir el índice, por lo que su selección debe estar apoyada en la teoría y el contexto.

Igualmente, es importante considerar que la selección del grupo de pertenencia de las variables es una decisión a priori de los investigadores, lo que implica que puede existir un sesgo en esta asignación. Asimismo, se debe tener en cuenta que las categorías con mayor número de variables tienden a influenciar el peso general del análisis, por lo que es importante construir grupos equilibrados.

\section{Clasificación no jerárquica alrededor de centros móviles}

Una vez construido el índice se crean grupos de riesgo para facilitar la interpretación de los resultados. Para ello se utiliza el método de centros móviles, que minimiza la varianza dentro de cada grupo conforme a la expresión que descompone la varianza total:

$\mathrm{I}=\sum \mathrm{p}_{\mathrm{q}} \mathrm{d}^{2}\left(\mathrm{~g}_{\mathrm{q}}-\mathrm{g}\right)+\sum_{\mathrm{q}} \sum_{\mathrm{i} \in \mathrm{q}} \mathrm{p}_{\mathrm{i}} \mathrm{d}^{2}\left(\mathrm{x}_{\mathrm{i}}-\mathrm{g}_{\mathrm{g}}\right)$

donde $\mathrm{g} \mathrm{y}_{\mathrm{q}}$ son los centros de gravedad de todos los individuos y de los individuos del grupo $q$ respectivamente $(\mathrm{q}=1, \ldots, \mathrm{k})$ y $\mathrm{p}\left(\mathrm{p}_{\mathrm{i}}\right.$ y $\mathrm{p}_{\mathrm{q}}$ son los pesos de los individuos dentro del grupo o en el total), $\mathrm{y} \mathrm{d}^{2}$ es el cuadrado de la distancia euclidiana. Así, a priori se determina número de segmentos $k$ a crear y se seleccionan arbitrariamente los centros iniciales de cada grupo. A continuación se hace una primera clasificación, asignando cada elemento al centro más cercano de acuerdo con una distancia euclidiana; luego se calcula el nuevo centro de cada segmento y se repite el proceso, que continúa hasta que no haya más 
cambios o la varianza entre grupos cambie en un valor inferior a un umbral dado.

Un inconveniente de este método es que los centros de gravedad se eligen inicialmente en forma arbitraria, por lo que la partición o segmentación obtenida es un óptimo local. Este problema tiende a corregirse iterando el algoritmo con diferentes centros iniciales y observando las particiones más estables.

\section{RESULTADOS}

Las variables y sus fuentes (cuadro 2) se dividen en cinco grupos de interés. El primer grupo corresponde a las variables relacionadas con la producción minera, y los otros cuatro corresponden a los ejes de conflicto descritos en la sección anterior (esquema 1).

Primero se construye un índice para cada explotación minera. Para ello es necesario depurar la base de datos para excluir municipios que no tienen explotación minera o de los que no se tiene información. Luego se realiza un primer ACP para comprobar que todas las variables tienen correlación positiva.

En el caso de las variables de ruralidad, índice de desempeño integral, necesidades básicas insatisfechas, índice de pobreza multidimensional, docentes por estudiante, colegios de nivel superior, mortalidad infantil y población afro, que tienen una correlación negativa con el resto de variables, se usa su complemento.

Esquema 1

Dimensiones del índice de conflicto por explotación de recursos naturales

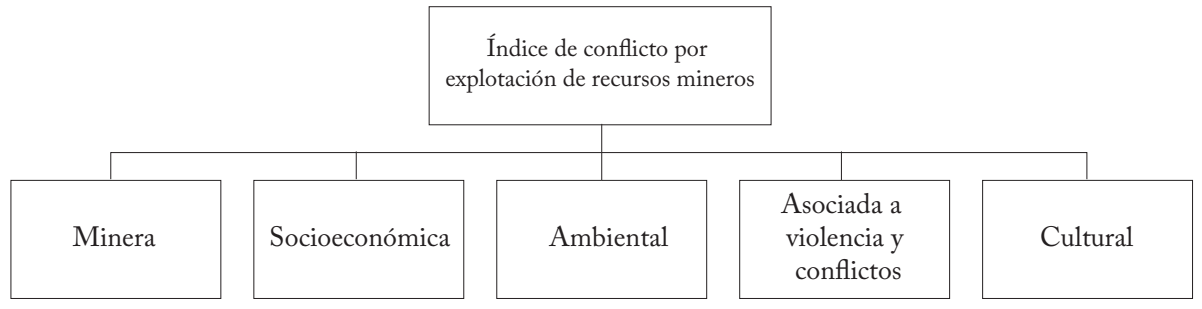

Fuente: elaboración propia.

En este punto se utiliza el AFM y se comprueba el peso de cada una de las variables en cada eje para escoger el eje al que más contribuye la variable de producción minera, y así construir un índice de conflictos relacionado con la explotación minera y no un índice de conflictos.

En el caso del carbón es necesario excluir las variables de sismo y desastres, pues no hay ningún caso en los 263 municipios productores. 
El eje 3 contiene la mayor contribución de la producción de carbón (cuadro 1), seguido por variables de salud como vacunación, minería de hecho, regalías, área del municipio, calidad del agua, porcentaje de población indígena y deslizamientos. A pesar de esto, no parece existir una alta correlación de las otras variables ambientales, sociales y de conflicto. Teniendo en cuenta lo señalado en las secciones segunda y tercera, una posible explicación es que aunque son municipios con pobres indicadores en estos aspectos, tienen niveles semejantes, por lo que no hay gran variabilidad, mientras que esto no sucede para las variables con alta contribución.

Unos 200 de los 263 municipios no tienen población indígena, y en los 63 restantes la participación es diversa, del 0,002\% al 1,53\%, con un coeficiente de variación ${ }^{4}$ del $141 \%$ entre estos municipios y de1 335\% cuando se considera toda la muestra.

En cuanto a la minería de hecho, en 199 municipios no se reporta caso alguno de minas informales o artesanales, mientras que en los 64 restantes existen reportes desde 1 a 78 minas, lo que implica un coeficiente de variación del $167 \%$ entre estos municipios y del $267 \%$ cuando se considera toda la muestra.

Por el contrario, la mortalidad infantil tiene coeficiente de variación del $46 \%$ en los municipios carboníferos, y la tasa de homicidios tiene un coeficiente de variación del 85\% en los 197 municipios que presentan valores, y del $127 \%$ cuando se considera toda la muestra.

Al hacer la clasificación se obtiene: 1 municipio en Muy alto, 4 en Alto, 3 en Medio, 33 en Bajo y 222 en Muy bajo. Los municipios de las tres primeras categorías corresponden a los que tienen grandes explotaciones mineras como La Jagua de Ibirico, Barrancas, Becerril, Chiriguaná, Hatonuevo, El Paso, Tumaco y Cúcuta. Estos resultados son intuitivos pues, como se señaló en las secciones 2 y 3 , la mayoría estos municipios se encuentra alrededor de las explotaciones de Drummond y E1 Cerrejón, a las cuales se señala por atraer conflictos en la zona.

En el caso del petróleo es necesario excluir las variables de sismos, contaminación, sequías, desastres naturales y volcanes, pues no se presenta ningún caso en 107 municipios con esta explotación. Se escoge el primer eje para la construcción de los pesos del índice por la gran contribución de la producción petrolera (cuadro 1). En este caso, población indígena, las variables de violencia, las variables ambientales (con excepción de calidad del agua) y las variables de regalías,

\footnotetext{
${ }^{4}$ El coeficiente de variación mide la variabilidad de una muestra y se calcula como la razón entre la desviación estándar y la media, multiplicada por 100.
} 
mortalidad infantil, minería de hecho, área del municipio y algunas de las variables de salud son las más relacionadas con este eje; mientras que transferencias, las variables de educación, la calidad del agua y la población afro no están tan relacionadas con esta problemática.

Mediante la clasificación se obtiene: 1 municipio en Muy alto, 1 en Alto, 7 en Medio, 13 en Bajo y 84 en Muy bajo. Los municipios de las tres primeras categorías corresponden a Puerto Gaitán, Acacias, Castilla La Nueva, Puerto Boyacá, Barrancabermeja, Arauca, Yopal, Aguazul y Orocué. Estos resultados son los esperados, pues incluyen municipios que han sido afectados por las diferentes conflictividades, como Puerto Gaitán, donde la problemática social se destaca por su rápido crecimiento, y Arauca, que enfrenta todos los problemas de seguridad alrededor de Caño Limón.

Puesto que el ferroníquel solo se explota en Montelíbano, su producción se une a la de oro para construir un índice y una clasificación conjunta. El tercer eje tiene la mejor representación conjunta de las dos variables mineras (cuadro 1); junto con la minería de hecho, la participación de comunidades indígenas, los homicidios y las tormentas son las que más aportan a este eje, por lo que tienen los comportamientos más disímiles entre los municipios productores de estos dos minerales metálicos.

De los 335 municipios que producen estos dos minerales, 3 están en categoría Muy alta, 9 en Alta, 17 en Media, 39 en Baja y 267 en Muy baja ${ }^{5}$. Estos resultados son de esperar, pues se sabe que en Segovia y Caucasia, por ejemplo, hay problemas en las comunidades por la minería artesanal, informal e industrial, que ha provocado violencia $\mathrm{y}$ afectaciones ambientales.

Por último se construye un indicador agregado de minería, para el cual se calcula la producción minera en miles de millones de pesos de 2015 como la suma de la producción de cada mineral ${ }^{6}$. Al excluir los municipios sin producción minera se obtienen 505 que producen alguno de los cuatro recursos. En este caso, la producción minera contribuye en un $61 \%$ a la construcción del tercer eje, seguida por regalías, homicidios, delitos sexuales, acciones unilaterales, inundación, tormenta, contaminación e incendio, y en menor medida, mortalidad infantil, cultivos de coca, delitos sexuales, entre otros. Cabe destacar que las comunidades indígenas y la minería de hecho tienen gran

${ }^{5}$ Los municipios de las dos primeras categorías son El Bagre, Segovia, Tarazá, Cáceres, Caucasia, Maceo, Remedios, Zaragoza, Istmina, Cumaribo, Santa Rita y Nóvita.

${ }^{6}$ También se hizo el ejercicio considerando cada variable de producción minera por separado, sin que hubiese cambios en los resultados. 
peso en los indicadores por separado, pero pierden peso en el indicador conjunto. Este comportamiento obedece a que al aumentar la muestra, algunas variables pierden variabilidad (al considerar un solo recurso su comportamiento es más homogéneo), mientras otros la ganan (al considerar toda la muestra, algunos municipios tienen comportamientos disímiles en comparación con su comportamiento frente a un recurso en particular).

En el caso de este índice existen muchos datos atípicos, es decir, que ocasionan que la clasificación por medias móviles forme grupos de uno o dos municipios. Unos 90 municipios arrojan mediciones muy altas ${ }^{7}, y$ como este número es muy alto se aplica la medida de Chebyshev que solo arroja 9 municipios atípicos ${ }^{8}$.

Existen 9 municipios en clasificación Muy alta, 8 en Alta, 12 en Media, 27 en Baja y 449 en Muy baja ${ }^{9}$. Estos resultados en algunos casos pueden ser contradictorios con los observados cuando se mide cada indicador por separado, pero en este caso también se analiza la interrelación entre los minerales, lo que puede elevar el riesgo en algunos municipios.

El cuadro 1 del anexo describe el peso de cada variable en el índice general, así como en los de carbón, oro, petróleo, oro y ferroníquel. Los mapas 1 a 4 muestran la intensidad de los índices de carbón, petróleo, oro y ferroníquel, y de la minería en general.

\section{CONCLUSIONES}

Este trabajo propone un índice de conflictividad asociada a la explotación minera para analizar las principales teorías desde una perspectiva cuantitativa. Se consideraron 4 dimensiones de conflictividad: social, ambiental, de conflicto y cultural. Se construyeron 3 indicadores para estudiar la conflictividad asociada a la explotación aurífera, carbonífera y petrolera. Y se construyó un indicador agregado, controlando por tamaño muestral.

E1 método permite hacer comparaciones entre municipios, pues estandariza variables con diferentes escalas de medición. Sin embargo, solo permite conocer correlaciones entre las variables, no relaciones de

\footnotetext{
${ }^{7}$ Un dato se considera atípico cuando se encuentra a más del cuartil 1 menos el recorrido intercuartil por 1,5 o al cuartil 3 más 1,5 por el recorrido intercuartil.

${ }^{8}$ Esto, siguiendo con los postulados de Chebyshev, quien demuestra que en cualquier distribución de momentos, un $95 \%$ de los datos se encuentra a 2 desviaciones estándar de la media.

${ }^{9}$ Los municipios de las dos primeras categorías son Puerto Boyacá, Acacías, Castilla la Nueva, Puerto Gaitán, Barrancabermeja, Arauca, Yopal, Aguazul, Orocué, Yondó, La Jagua de Ibirico, Neiva, Villavicencio, Melgar, Arauquita, Tauramena y Villagarzón.
} 
Mapa 1

Índice de conflicto, carbón

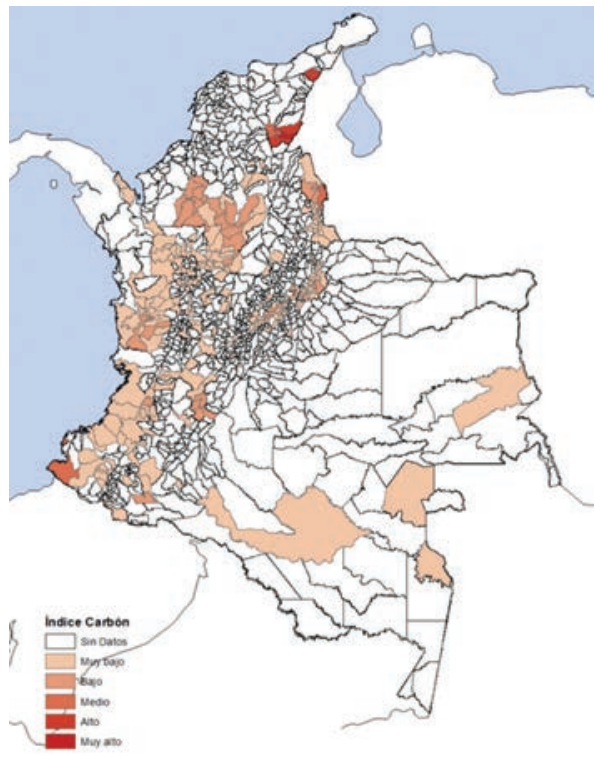

Fuente: elaboración propia.

Mapa 3

Índice de conflicto, oro y ferroníquel

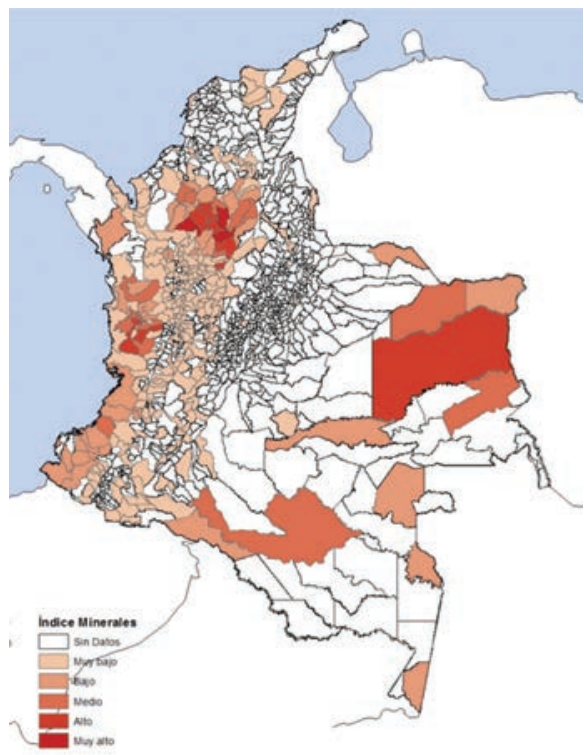

Fuente: elaboración propia.
Mapa 2

Índice de conflicto, petróleo

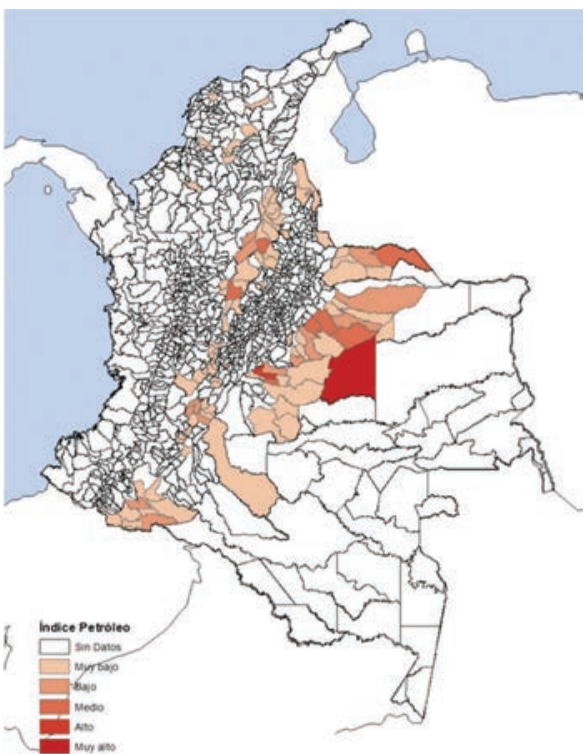

Fuente: elaboración propia.

Mapa 4

Índice de conflicto, minería

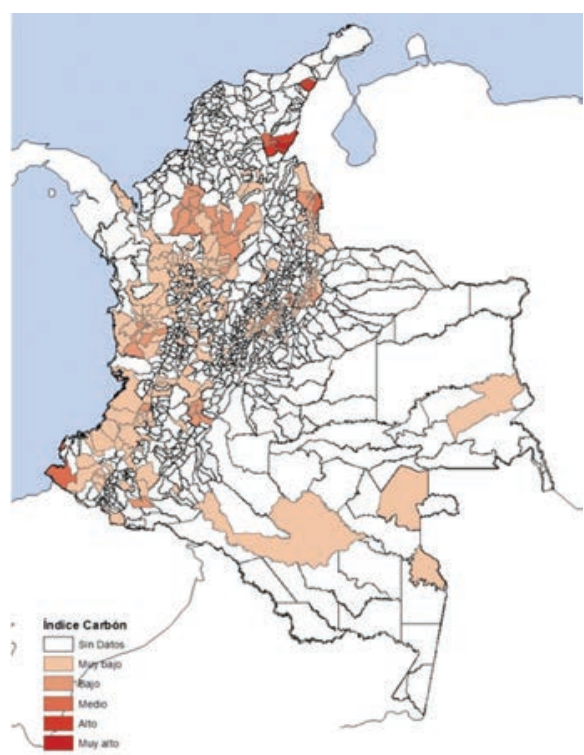

Fuente: elaboración propia. 
causalidad. Además, influyen visiblemente la variabilidad de los datos y la inclusión o exclusión de variables. No obstante, con respecto a la hipótesis de que no hay relación directa entre minería y conflicto, hay variación entre municipios. En todos los casos existen explotaciones mineras que son referentes de conflictividad asociada al tamaño de la explotación, como Puerto Gaitán, Montelíbano y La Jagua de Ibirico. Sin embargo, existen otras explotaciones que no parecen tener relación con los niveles de conflicto, como en la Zona Cafetera; por tanto, la hipótesis de Bannon y Collier (2003) no se cumple.

Con respecto a la hipótesis de que existe un efecto diferenciado entre explotación de recursos naturales y conflictividad, se encuentra que en las zonas carboníferas hay una correlación muy baja con la conflictividad regional. En cuanto a la explotación de petróleo, los resultados difieren entre municipios, en parte por sus condiciones propias, y en parte por el aporte de las variables al indicador. Sin embargo, la mayor conflictividad se concentra en pocas regiones que presentan los niveles de explotación más altos. Por último, los municipios con explotación de oro y ferroníquel presentan bajos niveles de conflictividad, pero las variables que más aportan al resultado son la presencia de minería artesanal e informal y comunidades indígenas, número de homicidios, y el conjunto de variables ambientales.

La tercera hipótesis establecía que aunque existe una relación entre la debilidad estatal y los conflictos en zonas de recursos naturales, esta no es una de las variables más correlacionadas con la explotación minera. Con el indicador agregado, los resultados muestran que las variables más relevantes, en cuanto a su correlación, para la existencia de conflicto son variables de salud, violencia, presencia de comunidades indígenas, variables ambientales, y cultivos ilegales, que son a su vez consecuencia de la baja presencia estatal y terminan generando conflictos económicos y sociales.

El estudio encuentra evidencia a favor de la hipótesis de que el desarrollo social es disímil entre las diferentes regiones de explotación minera. En efecto, los resultados de las estimaciones de los indicadores de forma independiente difieren de los resultados de la estimación del indicador conjunto, debido precisamente a la diversidad de estas regiones. Esto también se refleja en que los conflictos generados por la actividad minera no son iguales a lo largo de las zonas de explotación. 


\section{ANEXo}

\section{Cuadro 1}

Peso de las variables para el índice general

\begin{tabular}{|c|c|c|c|c|}
\hline Variable & $\begin{array}{l}\text { Îndice } \\
\text { general }\end{array}$ & Carbón & Petróleo & $\begin{array}{c}\text { Oro y } \\
\text { ferroníquel }\end{array}$ \\
\hline Producción mineral & 67,19 & & & \\
\hline Producción de carbón & & 53,36 & & \\
\hline Producción de petróleo & & & 30,05 & \\
\hline Producción de ferroníquel & & & & 0,15 \\
\hline Producción de oro & & & & 24,01 \\
\hline Minería de hecho & 0,52 & 3,55 & 2,16 & 19,69 \\
\hline Área del municipio, $\mathrm{km}^{2}$ & 0,45 & 0,13 & 2,94 & 0,86 \\
\hline Ruralidad & 0,02 & 0,00 & 0,34 & 0,14 \\
\hline Desempeño integral & 0,02 & 0,31 & 0,03 & 0,01 \\
\hline Necesidades básicas insatisfechas & 0,08 & 0,88 & 0,01 & 0,08 \\
\hline Índice de pobreza multidimensional & 0,12 & 0,66 & 0,14 & 0,08 \\
\hline Docentes por estudiante & 0,02 & 0,01 & 0,56 & 0,30 \\
\hline Colegios de nivel superior & 0,05 & 0,35 & 0,38 & 0,01 \\
\hline Mortalidad infantil & 0,87 & 0,36 & 1,10 & 0,19 \\
\hline Madres entre 15 y 19 años & 0,38 & 0,64 & 0,01 & 0,43 \\
\hline Polio & 0,43 & 4,70 & 1,26 & 0,27 \\
\hline DPT & 0,43 & 4,74 & 1,29 & 0,28 \\
\hline BCG & 0,15 & 3,08 & 1,65 & 0,21 \\
\hline Hepatitis B & 0,43 & 4,72 & 1,18 & 0,29 \\
\hline НIB & 0,43 & 4,74 & 1,29 & 0,29 \\
\hline $\mathrm{TV}$ & 0,38 & 4,23 & 1,16 & 0,35 \\
\hline Transferencias SGP & 0,01 & 0,24 & 0,11 & 0,35 \\
\hline Regalías & 6,37 & 4,11 & 5,14 & 0,03 \\
\hline Homicidios & 2,27 & 0,00 & 11,11 & 2,16 \\
\hline Hcoca & 0,01 & 0,15 & 1,21 & 0,26 \\
\hline Delitos sexuales & 1,06 & 0,01 & 2,24 & 0,10 \\
\hline Masacres & 0,20 & 0,11 & 3,56 & 0,13 \\
\hline Acciones unilaterales & 3,04 & 0,60 & 2,75 & 0,21 \\
\hline Daño a bienes civiles & 0,13 & 0,02 & 3,14 & 0,53 \\
\hline Sismo & 0,01 & & & 0,00 \\
\hline Inundación & 3,75 & 0,90 & 1,24 & 0,90 \\
\hline Contaminación & 2,11 & 0,05 & & 0,23 \\
\hline Incendio & 2,46 & 0,60 & 1,11 & 0,11 \\
\hline Deslizamiento & 1,24 & 1,46 & 0,09 & 0,00 \\
\hline Sequía & 1,07 & 0,11 & & 0,03 \\
\hline Desastre & 1,72 & & & 0,39 \\
\hline Tormenta & 2,21 & 0,02 & 1,71 & 1,69 \\
\hline Volcán & 0,13 & 0,41 & & 0,01 \\
\hline Calidad del agua & 0,09 & 2,07 & 0,61 & 0,87 \\
\hline Población indígena & 0,06 & 2,69 & 20,36 & 44,40 \\
\hline Población afro & 0,15 & 0,00 & 0,10 & 0,00 \\
\hline
\end{tabular}

Fuente: cálculos de los autores. 


\section{Cuadro 2 \\ Descripción y fuentes de las variables \\ De recursos mineros}

Producción de carbón, miles de millones de pesos de 2015: carbón producido entre 2005 y 2015, Sistema de Información Minero Colombiano (sımco)

Producción de petróleo, miles de millones de pesos de 2015: petróleo producido entre 2005 y 2015, Ministerio de Minas y Energía

Producción de oro, miles de millones de pesos de 2015: cantidad de oro producido entre 2001 y 2015, simco

Producción de ferroníquel, miles de millones de pesos de 2015: ferroníquel producido entre 2007 y 2015 , simco

Minería de hecho: explotaciones en minas ilegales, denuncias de las alcaldías municipales y la Personería de la Nación

Sociales y económicas

Área del municipio en $\mathrm{km}^{2}$, superficie del municipio, DANE

Ruralidad: porcentaje de población que vive fuera de la cabecera municipal, DANE

Desempeño integral del municipio: índice de desempeño integral del municipio de 2012, DNP

Necesidades básicas insatisfechas: porcentaje de hogares con al menos una necesidad básica insatisfecha en 2005, DANE

Índice de pobreza multidimensional: porcentaje de hogares en situación de pobreza multidimensional en 2005, DNP

Docentes por cada cien estudiantes en entidades oficiales; panel municipal del CEDE

Colegios de nivel superior y muy superior: colegios de nivel superior y muy superior de la clasificación ICFEs 11, panel municipal del CEDE

Mortalidad infantil: número de niños menores de un año muertos por cada cien mil nacidos vivos en 2013, DANE

Madres entre 15 y 19 años: número de mujeres que son madres entre 15 y 19 años en 2010, DANE Vacunación contra la polio: porcentaje de personas vacunadas contra la polio en $2010, \mathrm{Mi}^{-}$ nisterio de Salud

Vacunación contra DPT: porcentaje de personas vacunadas contra la DPT en 2010

Vacunación contra BCG: porcentaje de personas vacunadas contra la BCG en 2010, Ministerio de Salud

Vacunación contra hepatitis B: porcentaje de personas vacunadas contra la hepatitis B en 2010, Ministerio de Salud

Vacunación contra нів: porcentaje de personas vacunadas contra la нів en 2010, Ministerio de Salud

Vacunación contra TV: porcentaje de personas vacunadas contra la TV en 2010, Ministerio de Salud

Transferencias sgP: transferencias al Sistema General de Participaciones per cápita en 2012, DNP Y DANE

De violencia y conflicto

Homicidios: número de personas asesinadas por cada cien mil habitantes en 2012, Policía Nacional y DANE

Hectáreas de coca: superficie sembrada por municipio en 2012, Sistema Integrado de Monitoreo de Cultivos y Observatorio de Drogas

Delitos sexuales: número de delitos sexuales reportados en el municipio en 2012, Medicina Legal Masacres: número acumulado de masacres en el municipio desde 1980 hasta 2012, Memoria Histórica

Acciones unilaterales: número de acciones unilaterales (ataques, atentados, etc.) cometidas por un grupo armado en el municipio en 2012, Dirección de Investigación Criminal, Interpol y Oficina de Naciones Unidas para Asuntos Humanitarios (осHA)

Daños a bienes civiles: número acumulado de ataques a instituciones, a propiedad y a organizaciones, quema de vehículos, sabotaje y atentados por diferentes perpetradores en el periodo 1980-2012, Memoria Histórica

\section{Ambientales}

Sismos: número de sismos reportados en el municipio en 2013, оснA

Inundaciones: número de inundaciones reportadas en el municipio en 2013, осHA

Contaminación: número de casos de contaminación reportados, considerados como un desastre natural en el municipio en 2013, оснA 
Erosión: número de casos de erosión reportados en el municipio, en 2013, ocHA

Incendios: número de casos de incendio reportados en zonas naturales en el municipio en 2013, OCHA

Deslizamientos: número de deslizamientos reportados en el municipio en 2013, оснA

Sequías: número de sequías reportadas en el municipio en 2013, оснA

Tormentas: número de tormentas (vendavales, tormentas eléctricas, tornados, etc.) reportadas en el municipio en 2013, осHA

Volcanes: número de erupciones volcánicas reportadas en el municipio en 2013, осHA

Desastres: número de otros desastres naturales reportados en el municipio en 2013, ocHA

Calidad del agua: calidad del agua de las corrientes acuíferas del municipio, como porcentaje, Instituto de Hidrología, Meteorología y Estudios Ambientales (IDEAM)

\section{Culturales}

Población indígena: porcentaje de población indígena en el municipio sobre el total reportados en el país, Instituto Geográfico Agustín Codazzi (IgAc)

Población afrodescendiente: porcentaje de población afrodescendiente en el municipio sobre el total reportado en el país, IGAC

\section{Tratamiento de datos}

Aunque se hizo un gran esfuerzo para acopiar datos de todas las variables posibles, faltan medidas importantes en cada eje. Por ejemplo, efectos en el empleo, migración, afectaciones culturales, desaparición de comunidades, estrategias de mitigación de las empresas, corrupción. Para la minería se toman los reportes oficiales de producción, pero como la minería ilegal, artesanal y de otro tipo está extendida en el país, la base de datos no constituye un censo y puede ser una muestra sesgada.

Esa situación es muy probable en la explotación de oro y carbón, pues no requiere personal ni maquinaria especializada en la labor de extracción. Es menos probable en la extracción de petróleo debido a su alta tecnificación, e improbable en la de ferroníquel, pues hoy solo se explota una mina en el país. Por ello, en las variables de recursos mineros se incluye la minería de hecho para controlar el efecto no observado de este fenómeno.

Fuente: elaboración propia.

\section{REFERENCIAS BIBLIOGRÁFICAS}

1. Agencia Nacional de Hidrocarburos (2016). Historia del petróleo. Programa de Regionalización Sector Hidrocarburos. [http://www.anh.gov. co/portalregionalizacion/Paginas/Historia-del-petroleo-en-Colombia. aspx].

2. Akpalu, W. y Parks, P. J. (2007). Natural resource use conflict: Gold mining in tropical rainforest in Ghana. Environment and Development Economics, 12(1), 55-72.

3. AngloGold Ashanti (2015). Estudio de impacto ambiental 2015, Proyecto Minero Gramalote TM 14292 [https://issuu.com/anglogoldashanticolombia2/docs/eia_gramalote_anglogoldashanti_baja].

4. Bannon, I. y Collier, P. (2003). Natural resources and violent conflict: Options and actions. Washington: The World Bank.

5. Ввс (2010). El oro también financia a la guerrilla en Colombia. [http:// www.bbc.com/mundo/noticias/2010/11/101117_colombia_guerri1la_oro_financiacion_jg.shtml?print $=1]$.

6. Bebbington, A. (2009). Industrias extractivas, actores sociales y conflictos. A. Barandiarán. et al. (eds.), Extractivismo, politica y sociedad (pp. 131-156). Quito: cAAP y claes.

7. Bejarano, J. A. (1986). Efectos de la violencia en la producción agropecuaria. Misión de Estudios del Sector Agropecuario, Departamento Nacional de Planeación y Ministerio de Agricultura, Documento 6. 
8. Brunnschweiler, C. N. y Bulte, E. H. (2009). Natural resources and violent conflict: Resource abundance, dependence, and the onset of civil wars. Oxford Economic Papers, 61(4), 651-674.

9. Causey, R. L. (1969). Derived measurement, dimensions, and dimensional analysis. Philosophy of Science, 36(3), 252-270.

10. Chen, X. (2012). Social protest and contentious authoritarianism in China. Cambridge: Cambridge University Press.

11. Collier, P. (2007). El club de la miseria. Qué falla en los países más pobres del mundo. Madrid: Turner.

12. Cusaria, A. y Alfonso, J. (2004). Petróleo, ambiente y conflicto en Colombia. Guerra, sociedad y medio ambiente, 464-501.

13. De Echave, J.; Díez, A. et al. (2009). Minería y conflicto social. Lima: IEP y CIPCA.

14. De la Corte I. L. (2011). Criminalidad organizada y conflictos armados. Ejército de Tierra Española, 838, 18-26.

15. De la Hoz, J. V. (2009a). El ferroníquel de Cerro Matoso: aspectos económicos de Montelíbano y el Alto San Jorge. Banco de la República, documento de trabajo sobe Economía Regional y Urbana, 117.

16. De la Hoz, J. V. (2009b). Cerro Matoso y la economía del ferroníquel en el Alto San Jorge (Córdoba). Economía y Sociedad, 20, 41-58.

17. El Espectador (2014). La Colosa afectaría páramos del Tolima. [http:// www.elespectador.com/noticias/medio-ambiente/colosa-afectariaparamos-del-tolima-articulo-466736].

18. El Tiempo (2016). Trabajadores de Cerro Matoso deciden iniciar huelga. [http://www.eltiempo.com/economia/empresas/huelga-encerro-matoso/16607757].

19. El Tiempo (2016). Acuerdo evita huelga en Cerro Matoso (Córdoba). [http://www.eltiempo.com/colombia/otras-ciudades/evitan-huelga-encerro-matoso-cordoba/16621894].

20. Escoffier, B. y Pagés, J. (1992). Análisis factoriales simples y múltiples: objetivos, métodos e interpretaciones. Bilbao: Universidad del País Vasco.

21. Fairhead, J. (2001). International dimensions of conflict over natural and environmental resources. N. L. Peluso y M. Watts (eds.), Violent environments (pp. 213-236). Ithaca, Ny: Cornell University Press.

22. Fundación Ideas para la Paz (2014). La minera que quería tumbar el parque amazónico. [http://www.ideaspaz.org/tools/download/71507].

23. Foro Ciudades para la Vida (2002). Gestión comunitaria de riesgos, Primera parte. [http://www.minsa.gob.pe/ogdn/cd1/pdf/eri_04/parte1. pdf].\&\&

24. Goebertus, J. (2008). Oil palm and forced displacement in Zona Bananera: Pathways between natural resources and conflict. Colombia Internacional, 67, 152-175.

25. Gray, J. S. (1988). Centennial campaign: The sioux war of 1876 . Norman: University of Oklahoma Press.

26. Hernández, J. R. L. (2010). E1 petróleo en la historia económica colombiana. Pensamiento Humanista, 4, 52-63.

27. Humprhreys, M. (2005). Natural resources, conflict, and conflict resolution. Uncovering the Mechanisms. Columbia University. [http://www. columbia.edu/ mh2245/papers1/MH8JCR05_paper.pdf]. 
28. indepaz (2013). Petróleo. [http://ediciones.indepaz.org.co/wp content/ uploads/2013/06/Petroleo-Generalidades_de_la_industria_petrolera_en_Colobia-Revista_Indepaz_2013.pdf]

29. Jiménez, M. y Sideri, S. (1985). Historia del desarrollo regional en Colombia. Bogotá: cerec.

30. Le Dien, S. y Pagés, J. (2003a). Analyse factorielle multiple hiérarchique. Revue de Statistique Appliquée, 51(2), 47-73.

31. Le Dien, S. y Pagés, J. (2003b). Hierarchical multiple-factor analysis: Application to the comparison of sensory profiles. Food Quality and Preference, 14(5-6), 397-403.

32. Lebart, L.; Piron, M. et al. (2006). Statisitique exploratoire multidimensionnelle. París: Dunod.

33. Leiteritz, R.; Nasi, C. et al. (2009). Para desvincular los recursos naturales del conflicto armado en Colombia. Recomendaciones para formuladores de política y activistas. Colombia Internacional, 70, 215229.

34. Lewis, R. L. (1987). Black coal miners in America: Race, class, and community conflict, 1780-1980. Lexington: University Press of Kentucky.

35. Maxwell, K. (2004). Conflicts and conspiracies: Brazil and Portugal, 1750-1808. Londres: Routledge.

36. Mazo, C. A. (2013). Estudio documental sobre el papel de la minería ilícita como medio para el lavado de activos y financiación del terrorismo. [http://repository.unimilitar.edu.co/bitstream/10654/3393/2/ MazoRoldanCarlosAndres2011.pdf].

37. Mensah, S. O. y Okyere, S. A. (2014). Mining, environment and community conflicts: A study of company-community conflicts over gold mining in the Obuasi Municipality of Ghana. Journal of Sustainable Development Studies, 5(1), 64-99.

38. Monte, L. M.; Castellanos, M. G. et al. Bárbaros hoscos. Historia de resistencia y conflicto en la explotación del carbón en La Guajira, Colombia. Opera, 14, 47-69.

39. Morrice, E. y Colagiuri, R. Coal mining, social injustice and health: A universal conflict of power and priorities. Health $\mathcal{E}^{2}$ Place, 19, 74-79.

40. Morrison, K. (2010). Oil, conflict, and stability. Cornell University. [http://falcon.arts.cornell.edu/kmm368/Oil,\%20Conflict,\%20and\%20 Stability\%20July\%202010.pdf].

41. Ostler, J. (2004). The plains Sioux and US colonialism from Lewis and Clark to Wounded Knee. Nueva York: Cambridge University Press.

42. Özen, H. y Özen, Ş. Interactions in and between strategic action fields: A comparative analysis of two environmental conflicts in goldmining fields in Turkey. Organization E Environment, 24(4), 343-363.

43. Pagés, J. (2004). Multiple factor analysis: Main features and application to sensory data. Revista Colombiana de Estadistica, 27(1), 1-26.

44. Procuraduría General de la Nación (2011). Minería ilegal en Colombia. [https://www.procuraduria.gov.co/portal/media/file/MINERIA ILEGAL EN COLOMBIA DOCUMENTO.pdf].

45. Ramírez, J. G. y Muñoz, J. C. (2012). Informalidad e ilegalidad en la explotación del oro y la madera en Antioquia. Medellín: Universidad Eafit. 
46. Rettberg, A. y Ortiz, R. J. (2014). Conflicto dorado: explorando la relación entre minería de oro, conflicto armado y criminalidad en Colombia. Centro de Estudios sobre Seguridad y Drogas de la Universidad de los Andes.

47. Rivera, V. J. (2011). Análisis del impacto medio ambiental de El Cerrejón a partir de sus informes GRI: 2005-2009 (tesis de pregrado inédita). Pontificia Universidad Javeriana, Bogotá.

48. Rivera, L. et al. (2012). Minería, conflictos sociales y violación de derechos humanos en Colombia. Cinep/Programa por la Paz.

49. Rodríguez, G.; Peláez, M. et al. Inversión canadiense en Colombia: un análisis de las empresas extractivas. Revista de Economía del Caribe, $14,116-148$.

50. Rudas, G. (2014). Notas sobre la minería de carbón a gran escala en Colombia. Bogotá: Fundación Friedrich Ebert.

51. Rudas, G. y Espitia, J. (2013). La paradoja de la minería y el desarrollo. Análisis departamental y municipal para el caso colombiano. J. Garay y G. Rudas (eds.), La mineria en Colombia: institucionalidad y territorio, paradojas y conflictos (pp. 27-76). Bogotá: Contraloría General de la República.

52. Schueler, V.; Kuemmerle, T. et al. (2011). Impacts of surface gold mining on land use systems in Western Ghana. Ambio, 40(5), 528-539.

53. Scott, W. H. et al. (1963). Coal and conflict: A study of industrial relations at collieries. Liverpool: Liverpool University Press.

54. Semana (2012). Cerro Matoso: mina rica, pueblo pobre. [http:// www.semana.com/nacion/articulo/cerro-matoso-mina-rica-pueblopobre/262408-3].

55. Semana (2015). Cerro Matoso y gobierno realizan alianza por lo social para limpiar sus culpas. [http://sostenibilidad.semana.com/impacto/ articulo/cerro-matoso-gobierno-realizan-alianza-social-para-limpiarculpas/32673].

56. Shupe, M. C.; Wright, W. M. et al. (1980). Nationalization of the Suez Canal. A hypergame analysis. Journal of Conflict Resolution, 24(3), 477-493.

57. Torres, I. V. (2014). Dimensiones del extractivismo minero en Colombia. Análisis de las racionalidades de gobierno durante la última década. Análisis Politico, 27(82), 45.

58. Twose, N. (ed.) (1991). Greenwar. Londres: Panos.

59. Vásquez, H. (2012). La historia del petróleo en Colombia. Revista Universidad EAFIT, 30(93), 99-109.

60. Whiteman, G. y Mamen, K. (2002). Examining justice and conflict between mining companies and indigenous peoples: Cerro Colorado and the Ngabe-Bugle in Panama. Journal of Business and Management, 8(3), 293.

61. Woof, M. (2004). The dangers of illegal mining. World Mining Equipment, 3, 46. 